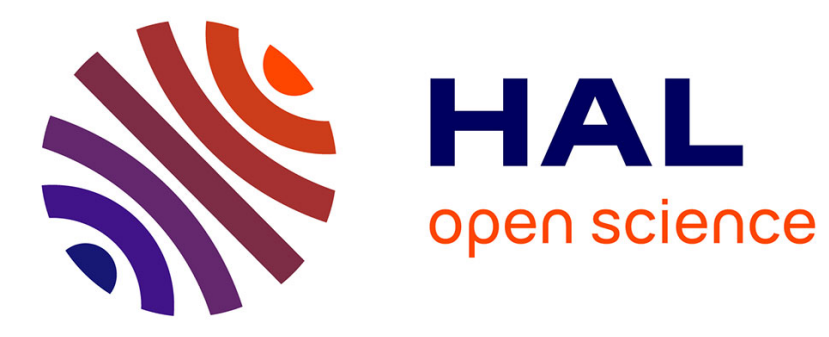

\title{
Bond orbital description of the strain-induced second-order optical susceptibility in silicon
} Pedro Damas, Delphine Marris-Morini, Eric Cassan, Laurent Vivien

\section{To cite this version:}

Pedro Damas, Delphine Marris-Morini, Eric Cassan, Laurent Vivien. Bond orbital description of the strain-induced second-order optical susceptibility in silicon. Physical Review B: Condensed Matter and Materials Physics (1998-2015), 2016, 93 (16), pp.165208. 10.1103/PhysRevB.93.165208 . hal01523525

\section{HAL Id: hal-01523525 \\ https://hal.science/hal-01523525}

Submitted on 16 May 2017

HAL is a multi-disciplinary open access archive for the deposit and dissemination of scientific research documents, whether they are published or not. The documents may come from teaching and research institutions in France or abroad, or from public or private research centers.
L'archive ouverte pluridisciplinaire HAL, est destinée au dépôt et à la diffusion de documents scientifiques de niveau recherche, publiés ou non, émanant des établissements d'enseignement et de recherche français ou étrangers, des laboratoires publics ou privés. 


\title{
Bond orbital description of the strain-induced second-order optical susceptibility in silicon
}

\author{
Pedro Damas, ${ }^{*}$ Delphine Marris-Morini, Eric Cassan, and Laurent Vivien ${ }^{\dagger}$ \\ Institut d'Electronique Fondamentale, Université Paris Sud, CNRS, UMR 8622, Université Paris-Saclay, Bât. 220, 91405 Orsay Cedex, France
}

(Received 11 November 2015; revised manuscript received 25 March 2016; published 26 April 2016)

\begin{abstract}
We develop a theoretical model, relying on the well established sp3 bond-orbital theory, to describe the strain-induced $\chi^{(2)}$ in tetrahedrally coordinated centrosymmetric covalent crystals, like silicon. With this approach we are able to describe every component of the $\chi^{(2)}$ tensor in terms of a linear combination of strain gradients and only two parameters $\alpha$ and $\beta$ which can be theoretically estimated. The resulting formula can be applied to the simulation of the strain distribution of a practical strained silicon device, providing an extraordinary tool for optimization of its optical nonlinear effects. The application of the first order theory to the photoelastic effect in $\mathrm{C}, \mathrm{Si}$, and $\mathrm{Ge}$ showed very good phenomenological and numerical agreement, up to $3 \% \mathrm{in} \mathrm{Si}$. The model was then used to the second-order nonlinear susceptibility, and we were able not only to confirm the main valid claims known about $\chi^{(2)}$ in strained silicon, but also estimate the order of magnitude of the $\chi^{(2)}$ generated in that device.
\end{abstract}

DOI: 10.1103/PhysRevB.93.165208

\section{INTRODUCTION}

Silicon-based photonics has generated a strong interest in recent years, mainly for optical communications and optical interconnects in CMOS circuits. The main motivations for silicon photonics are the reduction of photonic system costs and the increase of the number of functionalities on the same integrated chip by combining photonics and electronics, along with a strong reduction of power consumption [1]. However, one of the biggest constraints of silicon as an active photonic material is its vanishing second order optical susceptibility, the so-called $\chi^{(2)}$, due to the centrosymmetry of the silicon crystal. Without any second order nonlinear phenomena, fast and low power consumption optical modulation based on Pockels effect and wavelength conversions based on second harmonic generation (SHG) are not possible in bulk Si [2]. This is a very limiting factor when we expect silicon to be part of a solution to high performances and high energy efficient devices [3].

To overcome this problem, strain has been used as a way to deform the crystal and destroy the centrosymmetry which inhibits $\chi^{(2)}$. In fact, over the last few years Pockels electro-optic modulation [4-8] and SHG [9,10] have been claimed to be demonstrated in devices where the silicon active region is strained by a stress overlayer, usually made of SiN. Motivated by its enormous potential, the interest in strained silicon photonic devices has been growing in the past years. However, there is a lack of fundamental understanding on the process through which the strain tensor $\overline{\boldsymbol{\varepsilon}}$ generates nonvanishing $\chi^{(2)}$ tensor components. In other words, there is no available general quantitative relationship between these two quantities.

Despite the different attempts to find a solution to this problem, no theoretical model showing a practical relationship between the components of the second order nonlinear optical susceptibility $\chi^{(2)}$ and the strain tensor $\bar{\varepsilon}$ has been published yet. Such model is fundamental in the field of strained silicon photonics because it permits a connection between the strain

\footnotetext{
*pedro.damas@u-psud.fr

†laurent.vivien@u-psud.fr
}

effects, easy to simulate with the right computational tools, and the respective induced nonlinear phenomena. Without this knowledge, it is not possible to design and optimize structures based on strained silicon structures for the maximum outcome of $\chi^{(2)}$ effects.

The first proposed models connecting $\chi^{(2)}$ with $\bar{\varepsilon}[11,12]$ were based on the deformation potentials in semiconductors. The deformation potentials theory relies on the delocalization of the Bloch wave function over the entire crystal and proves to be a good description for the study of transport properties of electrons. However, when applied to the description of nonlinear optical phenomena, because it is not a vectorial theory, it proves to be very limiting in extracting and identifying the different $\chi^{(2)}$ tensor components in terms of $\bar{\varepsilon}$ and predict a good numerical agreement $[11,13]$.

Later, simpler models based on Coulomb interactions between atoms were also suggested [13,14], but none of them proved to a good description of the effects, both numerically and conceptually. To overcome this difficulty, $a b$ initio calculations were performed as an attempt to understand how the change in position of the atoms enables $\chi^{(2)}$ in the crystal $[9,15]$. Although they proved to be an accurate description of the problem, they are very computationally demanding and do not provide a practical, quantitative, and spatial relationship between $\chi^{(2)}$ and the strain $\overline{\boldsymbol{\varepsilon}}$ in the crystal and thus do not allow for device design over the strain distribution.

Even though the underlying process has not been described yet, it has been widely claimed that there is a direct relationship between $\chi^{(2)}$ and the strain gradients inside silicon [6-9,16]. In fact, very recently Manganelli et al. [17] proposed a model based on symmetry arguments to show a linear relationship between both the spatial distribution of $\chi^{(2)}$ and $\bar{\varepsilon}$ tensor components. However, even though the theory is well developed, it is very dependent on parameters required to be determined experimentally. This poses a problem because very recently it has been shown that the reported electro-optic measurements of strained silicon devices have a strong contribution from free carriers effects inside the silicon waveguide $[16,18,19]$. Therefore, most of the numerical data of strain-induced $\chi^{(2)}$ in silicon waveguides available in the literature was misinterpreted and discredited, making it impossible at the 
moment to find the experimental parameters predicted by the model presented in Ref. [17].

Because of its immense application potential, the theoretical description of nonlinear phenomena in covalent crystals has been the target of many different works in the past years. Some works suggest that a correct description of the nonlinear properties of solids must be addressed in a fully quantum-mechanical energy-band picture [20-22]. This is indeed the right approach for materials which have intrinsically nonzero bulk $\chi^{(2)}$. However, when $\chi^{(2)}$ is generated because of some localized effect where the crystal periodicity is locally perturbed, a local bond theory (such as the bond charge model [23] or the bond orbital model [24]) proves to describe very well the atomic nonlinear processes, from which tensor properties emerge automatically. In fact, the bond charge model was used to describe the surface-generated $\chi^{(2)}$ in silicon, where the crystal is abruptly terminated and $\chi^{(2)}$ is localized at the surface where the centrosymmetry is locally broken [25,26], which is conceptually very similar to what happens under strain.

Although many works have been reported, no consistent study based on the bond orbital model has been reported yet on the study of the strain-induced nonlinear effects in $\mathrm{Si}$. The bond orbital model is a widely accepted model to describe the local properties of electrons in the bonds of a tetrahedral covalent crystal like silicon [24,27,28]. Relying on the well established phenomenon of sp3-hybridization of silicon valence electrons, the properties of the bonding electrons are studied quantum mechanically. This procedure has already been used to characterize the strain effects [29] and nonlinear optical properties [27] in covalent crystals, although treated separately.

In the present paper, we rely on the original work first developed by Harrison et al. in Ref. [24] to describe the bonding electrons. By using the bond orbital model and keeping only first order effects in strain and strain gradients, we are able to show a practical way of knowing any $\chi^{(2)}$ component generated by the strain $(\bar{\varepsilon})$ in that point in space. The results not only show very good agreement with the main claims made in the literature about the relationship between $\chi^{(2)}$ and strain in silicon, but also depends only on two parameters which can be predicted theoretically and depend only on the material.

The organization of this paper is as follows: we start by presenting the general reasoning behind the model before entering into the quantum mechanical treatment of the sp3 hybridization in strained covalent crystals, in Sec. II.1. In Sec. II.3 we proceed to the calculation of the strain-induced polarity of a covalent bond, and in Sec. II.2, we deduce its second order nonlinear dipole moment. This will allow us to extract the strain-induced $\chi^{(2)}$ components in terms of the strain tensor $(\bar{\varepsilon})$ components. Finally, in Sec. IV we check the validity of our model by applying it to photoelasticity (a first-order effect) and study $\chi^{(2)}$ in a particular example device, showing the degree of the agreement and its practical applicability.

\section{THE STRAIN-INDUCED $\chi^{(2)}$ IN COVALENT CRYSTALS}

Silicon is a tetrahedral covalent crystal, where two neighbor atoms are bond together by sharing electrons, creating a co-

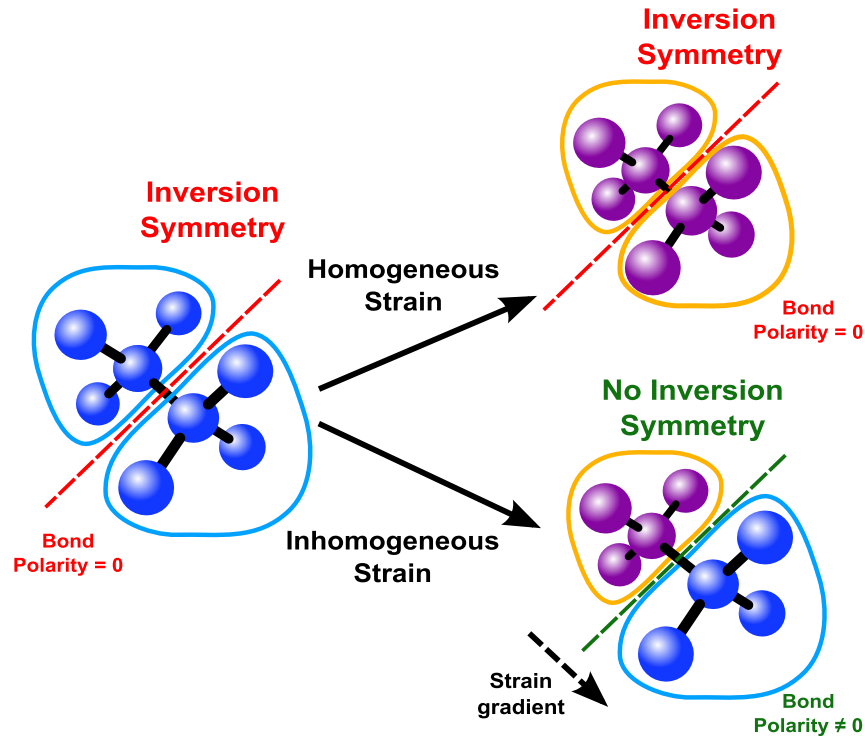

FIG. 1. The representation of the process of generation bond polarity, where different contour colours represent different electron energies. A homogeneous strain changes the energy of the bonding electrons, but it is still the same in both sides of the bond (yellow contours), keeping its inversion symmetry. However, a strain gradient, changes the energy in both sides of the bond (represented with the yellow and blue contours), creating a difference in energy polarity and destroying the inversion symmetry. The generated bond polarity is the origin of $\chi^{(2)}$ in that bond.

valent bond [27]. Four covalent bonds organize themselves in a tetrahedral configuration as shown in Fig. 1. Understanding any property of the crystal (like $\chi^{(2)}$ ) requires studying the quantum mechanical interactions of the electrons in the bonds. However, to make it clearer for the reader, before entering in any mathematical description, we start by presenting the process suggested in this paper for the generation of $\chi^{(2)}$ in each bond due to the strain.

It is known that $\chi^{(2)}$ depends primarily on the polarity of the bonds of a crystal [23,28,30]. The polarity of a bond is the difference of energy of the two electrons in the bond. In a centrosymmetric crystal, since the electrons feel the same energy in both directions, the bonds are unpolar, as shown in Fig. 1. However, when strain is applied to a crystal, the atomic configuration changes and a bond becomes polar if and only if there is a strain gradient in the direction of the bond. This process is presented schematically in Fig. 1, where the blue and yellow contours represent different values of energy. This explains why inhomogeneous strain fields are required to induce bond polarity and thus $\chi^{(2)}$, because homogeneous strain changes the electronic energy, but in the same way in both electrons of the bond.

This is the idea behind the mathematical and quantum description of our model: First we calculate how the strain changes the polarity of a bond and from there we deduce how that strain induces second order nonlinear effects. Moreover, even though in this paper we focus on silicon atoms, this procedure can be applied to any covalent diamond crystal structure. 
(a)

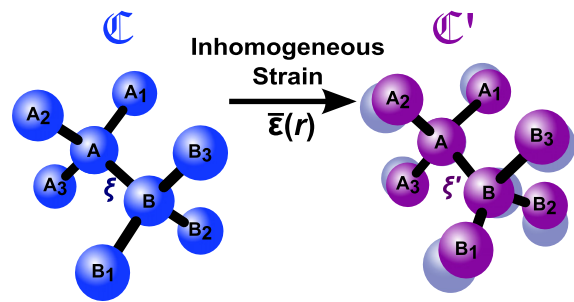

(b)

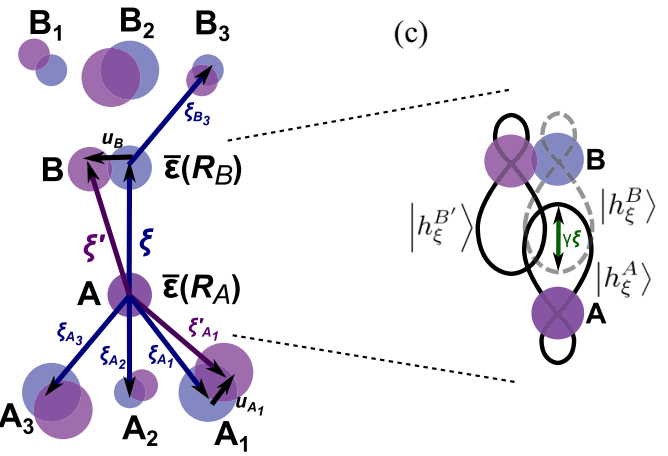

FIG. 2. (a) 3D Representation of the bond $\xi$ between the general atoms $A$ and $B$ in a unstrained silicon lattice $\mathfrak{C}$ unit cell, in blue. In purple is the corresponding structure when an inhomogeneous strain field is applied, generating the lattice $\mathfrak{C}^{\prime}$. (b) The 2D projection of the unit cells in (a), with the representation of the relevant vectors used in the text. (c) Schematic representation of the bond hybrids in the original and in the strained crystal.

\section{A. Quantum mechanical treatment of a strained covalent crystal}

Consider a tetrahedral covalent crystal $\mathfrak{C}$, represented in blue in the 3D scheme of Fig. 2(a). The four valence electrons in a general $\mathrm{Si}$ atom $A$ organize themselves in four different sp3 hybrids $\left|h_{\xi}^{A}\right\rangle$ pointing in the direction of the four nearest neighbors, creating four bonds $\xi(\xi=1,2,3,4)$. Such hybrid orbitals on any given atom are orthogonal to each other if the near neighbours are exactly tetrahedral, like in an unstrained Si crystal [24].

Consider now the lattice $\mathfrak{C}^{\prime}$ (represented in purple), the strained version of $\mathfrak{C}$. Each atom $n$ in $\mathfrak{C}^{\prime}$ is slightly moved by a vector $\boldsymbol{u}_{n}$ in relation to $\mathfrak{C}$ and the new atomic organization in $\mathfrak{C}^{\prime}$ will change a bond $\xi$ into $\xi^{\prime}$ [see Fig. 2(b)]. To study this new bonding, it may sound appealing to construct four new hybrids out of the atomic orbitals, pointing in the direction of $\xi^{\prime}$. However, since the atoms are not arranged in a tetrahedral configuration anymore, such a set of hybrids would not be orthogonal, and it would require a special treatment afterwards [31]. To overcome this problem, we will construct the wave function of the electrons in any atom $n$ as a combination of the original hybrids $\left|h_{\xi}^{n}\right\rangle$, placed in the new atomic positions. This is schematically represented in Fig. 2(c), making it possible to deal with the quantum mechanical subtleties of this problem, as will be apparent later on.

Consider now, without any loss of generality, atom $A$, taken as the atom which preserves the same position in $\mathfrak{C}^{\prime}$ and $\mathfrak{C}$, as shown in Figs. 2(a) and 2(b). This atom is connected to four other atoms. We focus on one of its four bonds, connecting atom $A$ with atom $B$ and call it bond $\xi$. The bond vector $\xi$ (associated with bond $\xi$ ) is defined as the vector from atom $A$ to atom $B$. [Fig. 2(b)].

To study the quantum mechanical properties of the electrons in the bond, we must start by building its Hamiltonian. The one-electron Hamiltonian of the strained crystal lattice $\mathfrak{C}^{\prime \prime}$ is given by [32]:

$$
H^{\prime}=T+\sum_{n} V_{n}^{\prime}=T+V_{A}^{\prime}+V_{B}^{\prime}+\sum_{n \neq A, B} V_{n}^{\prime}
$$

where $T$ is the kinetic energy of the electron and $V_{n}^{\prime}(\boldsymbol{r})=$ $V\left(\boldsymbol{r}-\boldsymbol{R}_{n}^{\prime}\right)$ is the potential due to the atom in position $\boldsymbol{R}_{n}^{\prime}$. This potential can be written as

$$
V_{n}^{\prime}(\boldsymbol{r})=V_{n}(\boldsymbol{r})+\Delta V_{n}(\boldsymbol{r}),
$$

with $V_{n}(\boldsymbol{r})=V\left(\boldsymbol{r}-\boldsymbol{R}_{n}\right)$ and $\Delta V_{n}$ being the contribution from the strain effects, vanishing for an unstrained crystal. We have explicitly separated $V_{A}^{\prime}$ and $V_{B}^{\prime}$ from the sum in Eq. (1) because we are focusing on the bond between atoms $A$ and $B$ and its treatment is more clear this way.

The matrix element of $H^{\prime}$ in $\left|h_{\xi}^{A}\right\rangle$ is given by

$$
\begin{aligned}
\left\langle h_{\xi}^{A}\left|H^{\prime}\right| h_{\xi}^{A}\right\rangle= & \left\langle h_{\xi}^{A}\left|T+V_{A}^{\prime}\right| h_{\xi}^{A}\right\rangle+\left\langle h_{\xi}^{A}\left|V_{B}^{\prime}\right| h_{\xi}^{A}\right\rangle \\
& +\sum_{n \neq A, B}\left\langle h_{\xi}^{A}\left|V_{n}^{\prime}\right| h_{\xi}^{A}\right\rangle .
\end{aligned}
$$

The corresponding expression is true for $\left|h_{\xi}^{B^{\prime}}\right\rangle$, which is the translation of the hybrid $\left|h_{\xi}^{B^{\prime}}\right\rangle$, pointing in the original direction of $B$ to $A$ in $\mathfrak{C}$, to the new location of atom $B$ in the strained crystal $\mathfrak{C}^{\prime}$, as shown in Fig. 2(c). We should now relate these matrix elements with ones of the unstrained Hamiltonian $H=$ $T+\sum_{n} V_{n}$ in the original hybrids basis. Since the hybrid wave functions $\left|h_{\xi}^{A}\right\rangle$ and $\left|h_{\xi}^{B^{\prime}}\right\rangle$ are, respectively, centered at the atoms $A$ and $B$ in $\mathfrak{C}^{\prime}$, it is clear that:

$$
\begin{aligned}
\left\langle h_{\xi}^{A}\left|T+V_{A}^{\prime}\right| h_{\xi}^{A}\right\rangle & =\left\langle h_{\xi}^{A}\left|T+V_{A}\right| h_{\xi}^{A}\right\rangle \\
\left\langle h_{\xi}^{B^{\prime}}\left|T+V_{B}^{\prime}\right| h_{\xi}^{B^{\prime}}\right\rangle & =\left\langle h_{\xi}^{B}\left|T+V_{B}\right| h_{\xi}^{B}\right\rangle
\end{aligned}
$$

Moreover, because of the symmetry of the bond, the potential of $A$ in $\left|h_{\xi}^{B^{\prime}}\right\rangle$ is the same as the potential of $B$ in $\left|h_{\xi}^{A}\right\rangle$. Thus,

$$
\left\langle h_{\xi}^{A}\left|V_{B}^{\prime}\right| h_{\xi}^{A}\right\rangle=\left\langle h_{\xi}^{B^{\prime}}\left|V_{A}^{\prime}\right| h_{\xi}^{B^{\prime}}\right\rangle=E_{A B}+\Delta E_{A B}
$$

where $E_{A B}=\left\langle h_{\xi}^{A}\left|V_{B}\right| h_{\xi}^{A}\right\rangle$ and $\Delta E_{A B}$ is the correction accounting for the new relative position of atoms $A$ and $B$ in $\mathfrak{C}^{\prime}$.

For all the other atoms $(n \neq A, B)$, we can write:

$$
\begin{aligned}
\left\langle h_{\xi}^{A}\left|V_{n}^{\prime}\right| h_{\xi}^{A}\right\rangle & =\left\langle h_{\xi}^{A}\left|V_{n}+\Delta V_{n}\right| h_{\xi}^{A}\right\rangle \\
& =\left\langle h_{\xi}^{A}\left|V_{n}\right| h_{\xi}^{A}\right\rangle+\left\langle h_{\xi}^{A}\left|\Delta V_{n}\right| h_{\xi}^{A}\right\rangle \\
\left\langle h_{\xi}^{B^{\prime}}\left|V_{n}^{\prime}\right| h_{\xi}^{B^{\prime}}\right\rangle & =\left\langle h_{\xi}^{B^{\prime}}\left|V_{n}+\Delta V_{n}\right| h_{\xi}^{B^{\prime}}\right\rangle \\
& =\left\langle h_{\xi}^{B}\left|V_{n}\right| h_{\xi}^{B}\right\rangle+\left\langle h_{\xi}^{B^{\prime}}\left|\Delta V_{n}\right| h_{\xi}^{B^{\prime}}\right\rangle
\end{aligned}
$$


From the previous analysis and after Eq. (3), we are in conditions of writing the matrix elements of $H^{\prime}$ as

$$
\begin{aligned}
\left\langle h_{\xi}^{A}\left|H^{\prime}\right| h_{\xi}^{A}\right\rangle & =E_{A}+\Delta E_{A B}+\sum_{n \neq A, B}\left\langle h_{\xi}^{A}\left|\Delta V_{n}\right| h_{\xi}^{A}\right\rangle \\
\left\langle h_{\xi}^{B^{\prime}}\left|H^{\prime}\right| h_{\xi}^{B^{\prime}}\right\rangle & =E_{B}+\Delta E_{A B}+\sum_{n \neq A, B}\left\langle h_{\xi}^{B^{\prime}}\left|\Delta V_{n}\right| h_{\xi}^{B^{\prime}}\right\rangle \\
\left\langle h_{\xi}^{A}\left|H^{\prime}\right| h_{\xi}^{B^{\prime}}\right\rangle & =U_{\xi}^{\prime} .
\end{aligned}
$$

The terms $\left\langle h_{\xi}^{A}|H| h_{\xi}^{A}\right\rangle=E_{A}$ and $\left\langle h_{\xi}^{B}|H| h_{\xi}^{B}\right\rangle=E_{B}$ are the average energy of $\left|h_{\xi}^{A}\right\rangle$ and $\left|h_{\xi}^{B}\right\rangle$ in $\mathfrak{C}$ and because of its centrosymmetry, they both have the same value $E_{A}=E_{B}$. The Hamiltonian crossterm $U_{\xi}^{\prime}$ is called the covalent energy.

\section{B. The optical dipole moment}

The nonlinear optical properties of the bond are extracted from its dipole moment, which in turn is calculated from the bond wave function. To do that, we now approach the problem as in the original theory done by Harrison et al. in Ref. [28]. The bond wave function $\left|b_{\xi}\right\rangle$ of the bond $\xi$ is considered to be a combination of the two adjacent hybrids of the atoms forming that bond [see Fig. 2(c)] [24,28,31], so we can write:

$$
\left|b_{\xi}\right\rangle=v_{A}\left|h_{\xi}^{A}\right\rangle+v_{B}\left|h_{\xi}^{B^{\prime}}\right\rangle
$$

and the coefficients $v_{A}$ and $v_{B}$ are obtained by minimizing the bond energy $E_{b}=\left\langle b_{\xi}\left|H^{\prime}\right| b_{\xi}\right\rangle /\left\langle b_{\xi} \mid b_{\xi}\right\rangle$. In doing so, we are implicitly neglecting all the other matrix elements of the Hamiltonian $H^{\prime}$ and all other hybrid overlaps are neglected (or absorbed in the parameters we have retained). It is important to bear in mind that $\left\langle h_{\xi}^{A} \mid h_{\xi}^{B^{\prime}}\right\rangle=S \neq 0$. Under these conditions, the explicit values for $v_{A}$ and $v_{B}$ can be found in the original work presented in Ref. [28].

The average position of the bond wave function $\left|b_{\xi}\right\rangle$ in a strained crystal, in respect to the center of the bond, can be shown to be given by $[28,33]$

$$
\langle\boldsymbol{r}\rangle=\left\langle b_{\xi}|\boldsymbol{r}| b_{\xi}\right\rangle=\left(v_{B}^{2}-v_{A}^{2}\right)\left[\frac{\boldsymbol{\gamma}_{\xi}}{2}\right]
$$

where $\boldsymbol{\gamma}_{\xi}=\boldsymbol{u}_{B}+\gamma \boldsymbol{\xi}$ and $\boldsymbol{u}_{B}$ is the displacement of atom B. In a nonstrained crystal $\langle\boldsymbol{r}\rangle$ reduces to $\left(v_{B}^{2}-v_{A}^{2}\right) \gamma \xi / 2$ which is the same result presented in Ref. [28]. In the original work, Harrison [28] introduces the parameter $\gamma$ which accounts for the distance between the "center of gravity" of each hybrid as shown in Fig. 2(c), and it would be unity if they were centered at the nucleus.

The optical effects are introduced by considering an optical electric field $\boldsymbol{E}$ that interacts with the bond and induces a dipole moment in it. Because the bond is made out of two electrons, its dipole moment is given by $\boldsymbol{p}_{\xi}=\langle\boldsymbol{p}\rangle_{\xi}=$ $-2 e\langle\boldsymbol{r}\rangle_{\xi}=-2 e\left\langle b_{\xi}|\boldsymbol{r}| b_{\xi}\right\rangle$, as represented in red in Fig. 3(a).

This optically-induced dipole moment changes the Hamiltonian by a term $\Delta H=\boldsymbol{p} \cdot \boldsymbol{E}=-2 e \boldsymbol{r} \cdot \boldsymbol{E}$, which must be included in the calculation of the bond wave function $\left|b_{\xi}\right\rangle$, resulting in coefficients $v_{A}$ and $v_{B}$ with some dependence on $\boldsymbol{E}$. The optically induced dipole moment in the bond will not only depend on $\boldsymbol{E}$ but also on the displacement $\boldsymbol{u}_{B}$, and the (a)

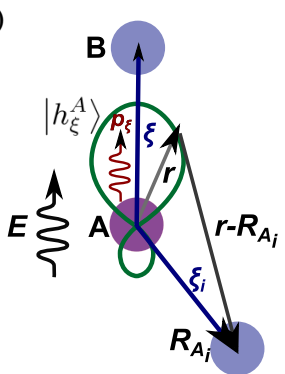

(b)

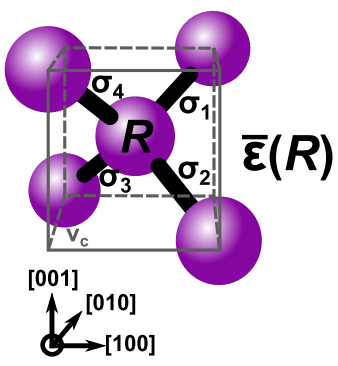

FIG. 3. (a) Representation of the optical electric field $\boldsymbol{E}$ generating the bond dipole moment $\boldsymbol{p}_{\boldsymbol{\xi}}$ and the vectors representation of the atomic position $\boldsymbol{R}_{A_{i}}$ of atom $A_{i}$ and electron position $\boldsymbol{r}$, useful for the integral in Eq. (35). (b) Silicon unit cell (with volume $v_{c}$ ) centered at the position $\boldsymbol{R}$ where each bond has its own polarity $\sigma_{i}$ induced by the strain tensor $\overline{\boldsymbol{\varepsilon}}(\boldsymbol{R})$ in the center of that unit cell.

resulting expression is

$$
\boldsymbol{p}_{\xi}=-2 e \frac{\sigma_{\xi}+\frac{e \boldsymbol{\gamma}_{\xi} \cdot \boldsymbol{E}}{2}}{\sqrt{U_{\xi}^{\prime 2}+\left(1-S^{2}\right)\left(\sigma_{\xi}+\frac{\left.e \boldsymbol{\gamma}_{\xi} \cdot \boldsymbol{E}\right)}{2}\right)^{2}}} .
$$

The quantity $\sigma_{\xi}$ is the so-called polar energy (or polarity) of the bond $\xi$ in the strained crystal and is defined by [24,28]

$$
\sigma_{\xi}=\frac{\left\langle h_{\xi}^{A}\left|H^{\prime}\right| h_{\xi}^{A}\right\rangle-\left\langle h_{\xi}^{B^{\prime}}\left|H^{\prime}\right| h_{\xi}^{B^{\prime}}\right\rangle}{2} .
$$

This is a measure of the energy difference in both sides of the bond and in a nonstrained centrosymmetric crystal it vanishes, i.e., $\sigma_{\xi}=0$.

Expanding the dipole moment in a Taylor series in the local optical field $\boldsymbol{E}$, yields $\left\langle\boldsymbol{p}_{\xi}\right\rangle=\boldsymbol{p}_{\xi}^{(0)}+\boldsymbol{p}_{\xi}^{(1)}+\boldsymbol{p}_{\xi}^{(2)}+\ldots$ where the different order terms are given by:

$$
\begin{aligned}
& \boldsymbol{p}_{\xi}^{(0)}=2 e\left[\frac{\sigma_{\xi}}{\left(U_{\xi}^{\prime 2}+\left(1-S^{2}\right) \sigma_{\xi}^{2}\right)^{\frac{1}{2}}}\right] \frac{\boldsymbol{\gamma}_{\xi}}{2} \\
& \boldsymbol{p}_{\xi}^{(1)}=2 e\left[\frac{U_{\xi}^{\prime 2}}{\left(U_{\xi}^{\prime 2}+\left(1-S^{2}\right) \sigma_{\xi}^{2}\right)^{\frac{3}{2}}}\right] \cdot\left(\frac{e \boldsymbol{\gamma}_{\xi} \cdot \boldsymbol{E}}{2}\right) \cdot \frac{\boldsymbol{\gamma}_{\xi}}{2} \\
& \boldsymbol{p}_{\xi}^{(2)}=-2 e\left[\frac{3}{2} \frac{\left(1-S^{2}\right) U_{\xi}^{\prime 2} \sigma_{\xi}}{\left(U_{\xi}^{\prime 2}+\left(1-S^{2}\right) \sigma_{\xi}^{2}\right)^{\frac{5}{2}}}\right] \cdot\left(\frac{e \boldsymbol{\gamma}_{\xi} \cdot \boldsymbol{E}}{2}\right)^{2} \cdot \frac{\boldsymbol{\gamma}_{\xi}}{2} .
\end{aligned}
$$

The $n$th order of the macroscopic polarization can be calculated from the previous equations by summing the contributions of the four bonds in the unit cell

$$
\boldsymbol{P}^{(n)}=\frac{1}{2 v_{c}} \sum_{\xi=1}^{4} \boldsymbol{p}_{\xi}^{(n)},
$$

where $v_{c}=2.01 \times 10^{-2} \mathrm{~nm}^{3}$ is the volume of the unit cell and the factor 2 accounts for the consideration of two electrons in the dipole moment of one bond.

Each order of the polarization has a specific role. The 0th order is related to the permanent polarization of the material and in bulk silicon it vanishes identically because $\sigma_{\xi}=0$. 
The 1st order polarization $\boldsymbol{P}^{(1)}$ is related to the dielectric permittivity of the material. The application of the bond-orbital model to this property of materials has shown very good numerical agreement with the experimental values [24,27]. Moreover, the strain effects on $\boldsymbol{P}^{(1)}$ corresponds to the photoelastic effect, which we shall analyze in Sec. IV.1 in order to validate the physical principles of our model.

Finally, $\boldsymbol{P}^{(2)}$ is related to the second order nonlinear optic effects, i.e., with $\chi^{(2)}$. Under no strain $\sigma_{\xi}=0$ and from Eq. (15) we see that $\boldsymbol{P}^{(2)}$ vanishes. This explains why there is no $\chi^{(2)}$ effects in centrosymmetric covalent crystals like $\mathrm{Si}$. Consequently, we must study the effects of strain on the polarity $\sigma_{\xi}$ to understand how we can make $\boldsymbol{P}^{(2)} \neq 0$.

\section{Strain-induced bond polarity}

The polarity of the bond $\xi$ defined in Eq. (12) in the strained crystal can be written as

$$
\begin{aligned}
\sigma_{\xi} & =\frac{\left\langle h_{\xi}^{A}\left|H^{\prime}\right| h_{\xi}^{A}\right\rangle-\left\langle h_{\xi}^{B^{\prime}}\left|H^{\prime}\right| h_{\xi}^{B^{\prime}}\right\rangle}{2} \\
& =\sum_{n \neq A, B} \frac{\left\langle h_{\xi}^{A}\left|\Delta V_{n}\right| h_{\xi}^{A}\right\rangle-\left\langle h_{\xi}^{B^{\prime}}\left|\Delta V_{n}\right| h_{\xi}^{B^{\prime}}\right\rangle}{2}
\end{aligned}
$$

and it can be immediately seen that if $\Delta V_{n}=0$, i.e., no strain is applied to the crystal, $\sigma=0$ and the bonds are nonpolar.

By reducing the sum in Eq. (12) only to the interaction between the first neighbors of atoms $A$ and $B$ individually, $A_{i}$ and $B_{i} i=1,2,3$, respectively, as shown in Figs. 2(a) and 2(b), Eq. (12) reduces to

$$
\sigma_{\xi}=\sum_{i=1}^{3} \frac{\left\langle h_{\xi}^{A}\left|\Delta V_{A_{i}}\right| h_{\xi}^{A}\right\rangle-\left\langle h_{\xi}^{B^{\prime}}\left|\Delta V_{B_{i}}\right| h_{\xi}^{B^{\prime}}\right\rangle}{2} .
$$

Despite that we have not said anything about the form of the crystal potential $V(\boldsymbol{r})$ yet, we know it is a central potential $[V(\boldsymbol{r})=V(r)]$ and for $r$ big enough, it should behave like a Coulomb potential. Therefore, for small displacements of the atoms, we may assume that $\left\|\boldsymbol{u}_{n}\right\| \ll\left\|\boldsymbol{r}-\boldsymbol{R}_{n}\right\|$ and the form of $\Delta V_{n}(\boldsymbol{r})$ defined in Eq. (2) can be taken by performing a first order Taylor expansion of the potential $V_{n}^{\prime}(\boldsymbol{r})$ :

$$
\begin{aligned}
V_{n}^{\prime}(\boldsymbol{r}) & =V\left(\boldsymbol{r}-\boldsymbol{R}_{n}^{\prime}\right)=V\left(\boldsymbol{r}-\left(\boldsymbol{R}_{n}+\boldsymbol{u}_{n}\right)\right) \\
& \sim V\left(\boldsymbol{r}-\boldsymbol{R}_{n}\right)-\nabla V\left(\boldsymbol{r}-\boldsymbol{R}_{n}\right) \cdot \boldsymbol{u}_{n} .
\end{aligned}
$$

By defining $\nabla V_{n} \equiv \nabla V\left(\boldsymbol{r}-\boldsymbol{R}_{n}\right)$, it is clear that

$$
\Delta V_{n}=-\nabla V_{n} \cdot \boldsymbol{u}_{n}
$$

which can only be evaluated once we know the explicit form of $V(\boldsymbol{r})$.

Using this definition along with the symmetries of the bond, the central properties of the potential $V(\boldsymbol{r})$ and bearing in mind that the hybrid wave functions satisfy $h_{\xi}^{B^{\prime}}(\boldsymbol{r})=h_{\xi}^{A}\left(\boldsymbol{R}_{B}^{\prime}-\boldsymbol{r}\right)$, it can be shown that

$$
\begin{aligned}
\left\langle h_{\xi}^{A}\left|\Delta V_{A_{i}}\right| h_{\xi}^{A}\right\rangle & =-\left\langle h_{\xi}^{A}\left|\nabla V_{A_{i}}\right| h_{\xi}^{A}\right\rangle \cdot \boldsymbol{u}_{A_{i}} \\
\left\langle h_{\xi}^{B^{\prime}}\left|\Delta V_{B_{i}}\right| h_{\xi}^{B^{\prime}}\right\rangle & =\left\langle h_{\xi}^{A}\left|\nabla V_{A_{i}}\right| h_{\xi}^{A}\right\rangle \cdot\left(\boldsymbol{u}_{B_{i}}-\boldsymbol{u}_{B}\right)
\end{aligned}
$$

leading to the simplification of Eq. (19) into

$$
\sigma_{\xi}=\sum_{i=1}^{3} \frac{\left\langle h_{\xi}^{A}\left|\nabla V_{A_{i}}\right| h_{\xi}^{A}\right\rangle \cdot\left(\boldsymbol{u}_{B}-\boldsymbol{u}_{B_{i}}-\boldsymbol{u}_{A_{i}}\right)}{2} .
$$

\section{Strain effects in a crystal}

The deformation state of a crystal is completely defined by the displacements $\boldsymbol{u}_{n}=\boldsymbol{u}\left(\boldsymbol{R}_{n}\right)$ in each atomic position $n$. In continuum mechanics, the local deformation at $\boldsymbol{R}_{n}$ is characterized by the so-called deformation gradient tensor $\overline{\boldsymbol{F}}$, whose components are defined by $[34,35]$ :

$$
F_{i j}=\frac{\partial u_{i}}{\partial x_{j}}+\delta_{i j} .
$$

From this definition, the displacement of any atom in $\boldsymbol{R}_{n}$ in respect to another atom in $\boldsymbol{R}_{0}$ is given by:

$$
\boldsymbol{u}_{n}=\boldsymbol{u}_{0}+\int_{\boldsymbol{R}_{0}}^{\boldsymbol{R}_{n}}(\overline{\boldsymbol{F}}(\boldsymbol{r})-\overline{\boldsymbol{I}}) \cdot d \boldsymbol{r},
$$

where $\overline{\boldsymbol{I}}$ is the identity matrix.

The previous integral is well defined and easy to evaluate in a continuous media, but in a crystal, where the position of the atoms are discrete, it requires a reinterpretation. Because the deformation is a small varying function over the bond length $d$ $\left(\frac{\partial F}{\partial x} . d<10^{-6}[17]\right)$, the adaptation of the problem to discrete positions can be done by tessellating the crystal into discrete elements and then considering that inside each element $\overline{\boldsymbol{F}}$ is constant [34-36].

For that reason, along the bonds $\boldsymbol{\xi}_{A_{i}}$ and $\boldsymbol{\xi}_{B_{i}}$ [where $\boldsymbol{\xi}_{A_{i}}$ is the bond vector between atoms $A$ and $A_{i}$ and, respectively, for $B$, as shown in Fig. 2(b)], the deformation gradient tensor can be considered to take the constant values $\overline{\boldsymbol{F}}\left(\boldsymbol{R}_{A}\right)$ and $\overline{\boldsymbol{F}}\left(\boldsymbol{R}_{B}\right)$, respectively. In that case, the integral in Eq. (23) directly gives

$$
\begin{gathered}
\boldsymbol{u}_{A_{i}} \simeq\left[\overline{\boldsymbol{F}}\left(\boldsymbol{R}_{A}\right)-\overline{\boldsymbol{I}}\right] \cdot \boldsymbol{\xi}_{A_{i}} \\
\boldsymbol{u}_{B_{i}} \simeq \boldsymbol{u}_{B}+\left[\overline{\boldsymbol{F}}\left(\boldsymbol{R}_{B}\right)-\overline{\boldsymbol{I}}\right] \cdot \boldsymbol{\xi}_{B_{i}} .
\end{gathered}
$$

In the case where the strain tensor has no shear components, i.e., $\varepsilon_{i j}=0$ for $i \neq j$ (and this is a critical condition) it is directly related to the deformation gradient tensor by $\overline{\boldsymbol{\varepsilon}}=\overline{\boldsymbol{F}}-$ $\overline{\boldsymbol{I}}$ [34-37]. Putting this relation in the previous equations allow us to relate the strain field in the crystal with the displacements of a atoms $A$ and $B$ :

$$
\begin{gathered}
\boldsymbol{u}_{A_{i}} \simeq \overline{\boldsymbol{\varepsilon}}\left(\boldsymbol{R}_{A}\right) \cdot \boldsymbol{\xi}_{A_{i}} \\
\boldsymbol{u}_{B_{i}} \simeq \boldsymbol{u}_{B}+\overline{\boldsymbol{\varepsilon}}\left(\boldsymbol{R}_{B}\right) \cdot \boldsymbol{\xi}_{B_{i}} .
\end{gathered}
$$

Putting together Eqs. (26) and (27) and bearing in mind that $\xi_{B_{i}}=-\boldsymbol{\xi}_{A_{i}}$ [Fig. 2(b)], Eq. (21) becomes

$$
\sigma_{\xi}=\frac{1}{2} \sum_{i=1}^{3}\left\langle h_{\xi}^{A}\left|\nabla V_{A_{i}}\right| h_{\xi}^{A}\right\rangle \cdot\left[\overline{\boldsymbol{\varepsilon}}\left(\boldsymbol{R}_{B}\right)-\overline{\boldsymbol{\varepsilon}}\left(\boldsymbol{R}_{A}\right)\right] \cdot \boldsymbol{\xi}_{A_{i}} .
$$

Since the strain changes slowly in distances of the bond length $d$, we can relate the $k l$ component of the strain tensor in atoms $A$ and $B$ with the strain gradient $\nabla \varepsilon_{k l}\left(\boldsymbol{R}_{A}\right)$ by making a first order Taylor expansion

$$
\varepsilon_{k l}\left(\boldsymbol{R}_{B}\right)=\varepsilon_{k l}\left(\boldsymbol{R}_{A}+\boldsymbol{\xi}\right) \sim \varepsilon_{k l}\left(\boldsymbol{R}_{A}\right)+\nabla \varepsilon_{k l}\left(\boldsymbol{R}_{A}\right) \cdot \boldsymbol{\xi} .
$$


We now define the rank-3 strain gradient tensor $\overline{\bar{\eta}}$ whose components are given by

$$
\eta_{i j k}=\frac{\partial \varepsilon_{i j}}{\partial x_{k}} .
$$

Using this definition and putting together Eqs. (29) and (28), we finally arrive at the relation between the strain gradients $\overline{\bar{\eta}}$ and the polarity of a bond $\xi$ in atom $A$ as

$$
\sigma_{\xi}(\overline{\overline{\boldsymbol{\eta}}})=\frac{1}{2} \sum_{i=1}^{3} \boldsymbol{\theta}_{i}^{\xi} \cdot \overline{\boldsymbol{\Xi}}_{\xi}(\overline{\overline{\boldsymbol{\eta}}}) \cdot \boldsymbol{\xi}_{A_{i}}
$$

where we have defined the rank-2 tensor $\overline{\boldsymbol{\Xi}}_{\xi}(\overline{\overline{\boldsymbol{\eta}}} ; \boldsymbol{R})$, related to the bond $\xi$, in the atom located in position $\boldsymbol{R}$ and with explicit dependence on the strain gradients $\overline{\bar{\eta}}$ in that position, given by

$$
\overline{\boldsymbol{\Xi}}_{\xi}(\overline{\overline{\boldsymbol{\eta}}} ; \boldsymbol{R})=\overline{\overline{\boldsymbol{\eta}}}(\boldsymbol{R}) \cdot \boldsymbol{\xi} .
$$

In addition, the vector $\boldsymbol{\theta}_{i}^{\xi}$ is defined by

$$
\begin{gathered}
\boldsymbol{\theta}_{i}^{\xi}=\left\langle h_{\xi}^{A}\left|\nabla V_{A_{i}}\right| h_{\xi}^{A}\right\rangle \\
=\int_{\infty}\left|h_{\xi}^{A}(\boldsymbol{r})\right|^{2} \cdot \nabla V\left(\boldsymbol{r}-\boldsymbol{R}_{A_{i}}\right) d V \\
=\left.\int_{\infty}\left|h_{\xi}^{A}(\boldsymbol{r})\right|^{2} \cdot \frac{\partial V}{\partial r}\right|_{\boldsymbol{r}-\boldsymbol{R}_{A_{i}}} \cdot \frac{\boldsymbol{r}-\boldsymbol{R}_{A_{i}}}{\left\|\boldsymbol{r}-\boldsymbol{R}_{A_{i}}\right\|} d V .
\end{gathered}
$$

Its evaluation can be done with the help of the scheme in Fig. 3(a). It does not depend on the atom $A$ in particular, but only on the unstrained bonds $\boldsymbol{\xi}_{i} \equiv \boldsymbol{\xi}_{A_{i}}, i=1,2,3$, which are the bonds in the unit cell different than $\xi$ [compare Fig. 3 with Fig. 2(b)].

The closed form of $\boldsymbol{\theta}_{i}^{\xi}$ can only be found once the potential $V(\boldsymbol{r})$ is known. However, regardless of that, we can always define

$$
\boldsymbol{\theta}_{i}^{\xi}=\alpha \boldsymbol{\xi}+\beta \boldsymbol{\xi}_{i}
$$

where $\alpha$ and $\beta$ are parameters whose values are related to the projections of $\boldsymbol{\theta}_{i}^{\xi}$ on $\boldsymbol{\xi}$ and $\boldsymbol{\xi}_{i}$, respectively, and are characteristic of the crystal species in consideration. This definition, together with Eq. (31), allows us to write

$$
\sigma_{\xi}(\overline{\overline{\boldsymbol{\eta}}})=\frac{1}{2} \sum_{i=1}^{3}\left[\alpha \boldsymbol{\xi}+\beta \boldsymbol{\xi}_{i}\right] \cdot \overline{\mathbf{\Xi}}_{\xi}(\overline{\overline{\boldsymbol{\eta}}}) \cdot \boldsymbol{\xi}_{i} .
$$

Expression (37) is the final expression for the polar energy (or polarity) of any bond $\xi$ in an atom centered in the unit cell located in $\boldsymbol{R}$ [see Fig. 3(b)]. The subscript $\xi$ identifies one of the four bonds, which defines the corresponding bond vector $\boldsymbol{\xi}$ and then the three other vectors $\boldsymbol{\xi}_{i}, i=1,2,3$. We see that it depends explicitly on the strain gradients through $\overline{\boldsymbol{\Xi}}_{\xi}(\overline{\overline{\boldsymbol{\eta}}} ; \boldsymbol{R})$, which is nonzero only if there is a strain gradient component in the direction of the bond $\xi$. This is relevant because it shows that in a centrosymmetric crystal not only a strain gradient is required to create a polar bond, but it also gives the preferred gradient direction to obtain maximum polarity in bond $\xi$.

Moreover, once the strain distribution $\bar{\varepsilon}(\boldsymbol{r})$ is known, the only parameters left to know are $\alpha$ and $\beta$. These two coefficients are the only unknowns of the model presented so far, and their value [defined in Eq. (36)] should be found experimentally, but this particular point requires further attention and it will be discussed later in Sec. IV.3.

\section{STRAIN DEPENDENT 2ND-ORDER NONLINEAR POLARIZATION}

The macroscopic nonlinear polarization $\boldsymbol{P}^{(\boldsymbol{n})}$ is an explicit local function of the strain and strain gradients, i.e. $\boldsymbol{P}^{(\boldsymbol{n})}(\overline{\boldsymbol{\varepsilon}}, \overline{\boldsymbol{\eta}} ; \boldsymbol{r})$. In fact, the covalent energy $U_{\xi}^{\prime}$ depends explicitly on strain $\overline{\boldsymbol{\varepsilon}}$ while the polarity $\sigma_{\xi}$ depends on the strain gradients $\bar{\eta}$. We focus now on the first order effects in $\overline{\boldsymbol{\varepsilon}}$ and $\bar{\eta}$ in the 2nd-order polarization.

Since $\boldsymbol{p}_{\xi}^{(2)}$ in Eq. (15) depends linearly on $\sigma_{\xi}(\overline{\boldsymbol{\eta}})$, the terms proportional to $\bar{\varepsilon}$ in that equation will introduce terms of the form $\varepsilon . \eta$, i.e., second order in strain effects. Therefore, in a first order theory, we must neglect the strain contributions from the other parameters, i.e., $U_{\xi}^{\prime}=U$ and $\boldsymbol{\gamma}_{\xi}=\gamma \boldsymbol{\xi}$. Moreover, $U$ is much bigger than the rather small strain-induced polarity of the bond, $\sigma_{\xi}$. All these considerations together with Eq. (16) for $n=2$ lead to

$$
\boldsymbol{P}^{(2)}=\frac{1}{2 v_{c}} \sum_{\xi=1}^{4} \boldsymbol{p}_{\xi}^{(2)}=-K \sum_{\xi=1}^{4} \sigma_{\xi}(\boldsymbol{\xi} \cdot \boldsymbol{E})^{2} \cdot \boldsymbol{\xi},
$$

where

$$
K=\frac{3}{2 v_{c}}\left(\frac{e \gamma}{2 U}\right)^{3}\left(1-S^{2}\right)
$$

Using the corresponding values for $\mathrm{Si}$, taking $\gamma=1.4$ [27] and $S=0.5$ [28], we have $K=1.18 \times 10^{29} \mathrm{C}^{3} \mathrm{~m}^{-3} \mathrm{eV}^{-3}$.

For a bond length $d$, the bond vectors $\xi$ in the crystal coordinates $\{\hat{\mathbf{1}}, \hat{\mathbf{2}}, \hat{\mathbf{3}}\}=\{[100],[010],[001]\}$ are given by

$$
\begin{aligned}
& \xi_{1}=\frac{d}{\sqrt{3}}(1,1,1), \quad \xi_{2}=\frac{d}{\sqrt{3}}(1,-1,-1) \\
& \xi_{3}=\frac{d}{\sqrt{3}}(-1,1,-1), \quad \xi_{4}=\frac{d}{\sqrt{3}}(-1,-1,1),
\end{aligned}
$$

and replacing these coordinates in Eq. 38, we can write the final components of the 2 nd order nonlinear polarization in the crystal coordinates as:

$$
\begin{aligned}
P_{x}= & \frac{K d^{3}}{3 \sqrt{3}}\left[\left(\sigma_{1}+\sigma_{2}-\sigma_{3}-\sigma_{4}\right)\left(E_{x}^{2}+E_{y}^{2}+E_{z}^{2}\right)\right. \\
& +2\left(\sigma_{1}-\sigma_{2}+\sigma_{3}-\sigma_{4}\right) E_{x} E_{y}+2\left(\sigma_{1}-\sigma_{2}-\sigma_{3}+\sigma_{4}\right) \\
& \left.\times E_{x} E_{z}+2\left(\sigma_{1}+\sigma_{2}+\sigma_{3}+\sigma_{4}\right) E_{y} E_{z}\right] \\
P_{y}= & \frac{K d^{3}}{3 \sqrt{3}}\left[\left(\sigma_{1}-\sigma_{2}+\sigma_{3}-\sigma_{4}\right)\left(E_{x}^{2}+E_{y}^{2}+E_{z}^{2}\right)\right. \\
& +2\left(\sigma_{1}+\sigma_{2}-\sigma_{3}-\sigma_{4}\right) E_{x} E_{y}+2\left(\sigma_{1}-\sigma_{2}-\sigma_{3}+\sigma_{4}\right) \\
& \left.\times E_{y} E_{z}+2\left(\sigma_{1}+\sigma_{2}+\sigma_{3}+\sigma_{4}\right) E_{x} E_{z}\right] \\
P_{z}= & \frac{K d^{3}}{3 \sqrt{3}}\left[\left(\sigma_{1}-\sigma_{2}-\sigma_{3}+\sigma_{4}\right)\left(E_{x}^{2}+E_{y}^{2}+E_{z}^{2}\right)\right. \\
& +2\left(\sigma_{1}+\sigma_{2}-\sigma_{3}-\sigma_{4}\right) E_{x} E_{z}+2\left(\sigma_{1}-\sigma_{2}+\sigma_{3}-\sigma_{4}\right) \\
& \left.\times E_{y} E_{z}+2\left(\sigma_{1}+\sigma_{2}+\sigma_{3}+\sigma_{4}\right) E_{x} E_{y}\right] .
\end{aligned}
$$


The previous set of equations, together with the definition of $\chi^{(2)}$ when a static electric field $E^{D C}$ is involved (the case of Pockels effect) [17],

$$
P_{i}^{(2)}=2 \epsilon_{0} \chi_{i j k}^{(2)} E_{j} E_{k}^{D C}
$$

determines every and each component of the $\chi^{(2)}$ tensor in the crystal coordinates in terms of the polarity of each bond $\sigma_{\xi}$ in the unit cell, as represented in Fig. 3(b). This polarity depends on the sum of the strain gradients projected on the direction of each bond, as shown by Eq. (32), leading to a different polarity of each bond in the unit cell. Therefore, in general, every component of $\chi^{(2)}$ will be nonzero, contrasting with the case of a zinc-blend crystal where each bond has the same polarity $\sigma$ and in which case Eqs. (41)-(43) lead to the well known fact that $\chi_{x y z}^{(2)}$ is the only nonvanishing component.

The explicit calculation of the $\chi^{(2)}$ components in terms of the strain gradients requires an explicit expansion of the sum defining $\sigma_{\xi}$ in Eq. (37). Despite being always possible to do it in terms of the unknown parameters $\alpha$ and $\beta$, we will not write it explicitly for a general case because it turns out to be a very big expression and it can be easily calculated in specific for the required situation. For that reason, we will rather apply it to a relevant device where we can actually analyze how the strain enables its $\chi^{(2)}$ effects.

\section{EVALUATION OF THE PROPOSED MODEL}

To fully validate the model presented in the previous sections, an experimental confirmation would be required. However, as already mentioned in the introduction, very recently it has been shown that the experimental data available in the literature on $\chi^{(2)}$ phenomena in strained silicon has a strong not totally understood contribution from free carriers effects $[16,18,19]$. This parasitic response masks the real value of strain-induced $\chi^{(2)}$ in silicon, resulting in erroneous experimental data. As a result, most of the quantitative values of $\chi^{(2)}$ in strained silicon presented in the literature [5-8] have been discredited, and no reliable data is available to confidently compare with the results from our model.

For that reason, we will check the validity of our model by applying it to the photoelastic effect, which has been widely studied in Group IV materials like C, Si, and Germanium. This verification is considered as a strong indicator that our model is able to describe the interaction between optical and strain effects in tetrahedral covalent crystals. In addition to this, we may also apply our model to some the structural characteristic of strained-induced $\chi^{(2)}$ in silicon, which have been verified experimentally.

\section{A. Photoelastic effect}

The dielectric tensor $\overline{\boldsymbol{\epsilon}}$ is related to the first-order optical susceptibility $\bar{\chi}^{(1)}$ by

$$
\overline{\boldsymbol{\epsilon}}=\overline{\boldsymbol{I}}+\bar{\chi}^{(1)} .
$$

When a cubic crystal is strained, the normally isotropic dielectric tensor becomes anisotropic. For small deformations, the linear relationship between the change in the inverse dielectric tensor $\delta\left(\overline{\boldsymbol{\epsilon}}^{-1}\right)$ and the strains form the photoelastic tensor $p_{i j k l}$, and it is often described by

$$
\delta\left(\overline{\boldsymbol{\epsilon}}^{-1}\right)_{i j}=p_{i j k l} \varepsilon_{k l} .
$$

The photoelastic effects directly arise from the strain dependence of $\bar{\chi}^{(1)}(\varepsilon)$ :

$$
\overline{\boldsymbol{\epsilon}}(\varepsilon)=\overline{\boldsymbol{I}}+\overline{\boldsymbol{\chi}}^{(1)}(\varepsilon) \simeq \overline{\boldsymbol{\epsilon}}+\Delta \overline{\boldsymbol{\epsilon}}(\varepsilon) .
$$

The first order expansion of $\bar{\chi}^{(1)}$ in $\varepsilon_{i j}$, provides us with the photoelastic tensor components [38]:

$$
\delta\left(\overline{\boldsymbol{\epsilon}}^{-1}\right)=p_{i j k l} \varepsilon_{k l}=-\frac{1}{n^{4}} \Delta \overline{\boldsymbol{\epsilon}}(\varepsilon),
$$

where $n$ is the refractive index of the material.

In cubic crystals, there are three independent photoelastic coefficients. In the compressed Voigt notation for symmetric tensors, those are [39]: $p_{11}, p_{12}$, and $p_{44}$. In this paper we focus only on the first two coefficients because $p_{44}$ relates the shear components of the strain, which we may not consider in our model. The calculation of $\bar{\chi}^{(1)}(\varepsilon)$ is obtained through the evaluation of $\boldsymbol{p}^{(1)}$ in Eq. (14) because

$$
\boldsymbol{P}^{(1)}=\epsilon_{0} \bar{\chi}^{(1)} \cdot \boldsymbol{E},
$$

which has a direct dependence on $\overline{\boldsymbol{\varepsilon}}$ through $U_{\xi}(\overline{\boldsymbol{\varepsilon}})$.

To study the photoelastic effect, we consider a homogeneous strain. In that case $\overline{\overline{\boldsymbol{\eta}}}=0, \sigma_{\xi}=0$, and $\boldsymbol{\gamma}_{\xi}=(\gamma \overline{\boldsymbol{I}}+\overline{\boldsymbol{\varepsilon}})$. $\xi$. Consequently, putting together Eqs. (14) and (16) for $n=1$ yields

$$
\boldsymbol{P}^{(1)}=\frac{e^{2}}{2 v_{c}} \sum_{\xi=1}^{4} \frac{\boldsymbol{\xi} \cdot(\gamma \overline{\boldsymbol{I}}+\overline{\boldsymbol{\varepsilon}}) \cdot \boldsymbol{E}}{U_{\xi}(\overline{\boldsymbol{\varepsilon}})} \cdot[(\gamma \overline{\boldsymbol{I}}+\overline{\boldsymbol{\varepsilon}}) \cdot \boldsymbol{\xi}] .
$$

The difficulty in evaluating the previous expression is in the explicit dependence of $U_{\xi}(\overline{\boldsymbol{\varepsilon}})$ on both the bond direction and strain effects. In general, the explicit form of $U_{\xi}(\overline{\boldsymbol{\varepsilon}})$ is not known but for some special cases, it can be estimated.

In this analysis we are mainly interested in checking the validity of the previous expression for $\boldsymbol{P}^{(1)}$ in a phenomenological way, i.e., check whether the photoelastic tensor properties for cubic crystals match with those resulting from Eq. (50) when first order strain effects are considered. A quantitative estimation of the photoelastic coefficients deeply depends on the correct expression for $U_{\xi}(\bar{\varepsilon})$, which is not a simple task, and best results are achieved by considering multiple potential contributions in computational simulations [38]. To deal with this problem, we select strain types where $U_{\xi}(\overline{\boldsymbol{\varepsilon}})$ is the same in every bond, so that the previous sum is simplified. We selected two situations where this occurs: uniaxial strain and the elastic angle distortion strain.

\section{Uniaxial strain}

Under uniaxial strain in the [001] direction, the only nonvanishing component of the strain tensor is $\varepsilon_{z z}=\varepsilon$. In this case, the strain makes the bonds undergo the same type of deformation in respect to each other and the covalent energy changes evenly in all bonds. Therefore $U_{\xi}(\overline{\boldsymbol{\varepsilon}})=U(\varepsilon)$ and from Eq. (50), the linear susceptibility $\bar{\chi}^{(1)}$ takes the form

$$
\bar{\chi}^{(1)}(\varepsilon)=\frac{d^{2} e^{2}}{3 \epsilon_{0} v_{c} U(\varepsilon)}\left(\begin{array}{ccc}
\gamma^{2} & 0 & 0 \\
0 & \gamma^{2} & 0 \\
0 & 0 & (\gamma+\varepsilon)^{2}
\end{array}\right) .
$$


TABLE I. Comparison between the calculated and experimental values of the photoelastic constants for $\mathrm{Si}$, C, and Ge. The two strain types studied in this work are compared with the respective experimental values.

\begin{tabular}{|c|c|c|c|c|c|c|c|}
\hline & \multicolumn{2}{|c|}{ Parameters } & \multicolumn{3}{|c|}{ Experimental } & \multicolumn{2}{|c|}{ Calculated } \\
\hline & $U(\mathrm{eV})$ & $\gamma$ & $p_{11}$ & $p_{12}$ & $p_{11}-p_{12}$ & $p_{11}$ (Uniaxial strain) & $p_{11}-p_{12}$ (Angle distortion) \\
\hline $\mathrm{C}$ & 6.94 & $1.13[27]$ & -0.249 & 0.043 & $-0.292[40,41]$ & -0.210 & -0.251 \\
\hline $\mathrm{Si}$ & 2.98 & $1.4[27]$ & -0.094 & 0.017 & $-0.111[38,41,42]$ & -0.095 & -0.108 \\
\hline $\mathrm{Ge}$ & 2.76 & $1.6[27]$ & -0.154 & -0.126 & $-0.030[43]$ & -0.196 & -0.070 \\
\hline
\end{tabular}

Expanding this expression in first order in $\varepsilon$ and using Eq. (48), it results in the following dielectric tensor change

$$
\delta\left(\overline{\boldsymbol{\epsilon}}^{-1}\right)=-\frac{d^{2} e^{2}}{3 \epsilon_{0} v_{c} n^{4} U^{2}}\left(\begin{array}{ccc}
\gamma^{2} \widehat{U} & 0 & 0 \\
0 & \gamma^{2} \widehat{U} & 0 \\
0 & 0 & \gamma(\gamma \widehat{U}-2 U)
\end{array}\right) \varepsilon,
$$

where $\widehat{U}=\left.\frac{\partial U}{\partial \varepsilon}\right|_{\varepsilon=0}$. The same tensor in terms of the photoelastic coefficients, for this type of strain, is given by [39]

$$
\delta\left(\overline{\boldsymbol{\epsilon}}^{-1}\right)=\left(\begin{array}{ccc}
p_{12} & 0 & 0 \\
0 & p_{12} & 0 \\
0 & 0 & p_{11}
\end{array}\right) \varepsilon .
$$

Comparing Eqs. (52) and (53) shows that $\delta\left(\overline{\boldsymbol{\epsilon}}^{-1}\right)$ has the same shape, and this shows that the strain dependent polarization deduced from our model has the symmetries expected for a cubic crystal. The corresponding photoelastic coefficients are

$$
\begin{gathered}
p_{11}=\frac{d^{2} e^{2} \gamma(2 U-\gamma \widehat{U})}{3 \epsilon_{0} v_{c} n^{4} U^{2}} \\
p_{12}=-\frac{d^{2} e^{2} \gamma^{2} \widehat{U}}{3 \epsilon_{0} v_{c} n^{4} U^{2}} .
\end{gathered}
$$

In spite of not knowing the value of $\widehat{U}$, we may check the validity of the previous equations by estimating $\widehat{U}=$ $\frac{3 p_{12} \epsilon_{0} v_{c} n^{4} U^{2}}{d^{2} e^{2} \gamma^{2}}$ from the measured value of $p_{12}$ and then check if inserting it in Eq. (55) results in the right value for $p_{11}$. We did so for diamond (C), silicon ( $\mathrm{Si}$ ), and germanium (Ge), and the results are presented in Table I. It is clear the very good agreement between experimental $p_{11}$ and the corresponding calculated value for the uniaxial strain, particularly for $\mathrm{Si}$, whose error is less than $3 \%$. The shape of $\delta\left(\overline{\boldsymbol{\epsilon}}^{-1}\right)$ in Eq. (52) together with the excellent numerical relation between $p_{11}$ and $p_{12}$ for three different tetrahedral centrosymmetric crystals, suggest that strain effects are well described by our model for this type of material.

\section{Elastic angle distortion strain}

This type of strain is characterized by no variation in bond length to first order, and all the energy change is due to an angular distortion of the atoms. The nonzero components of such kind of strain are $\varepsilon_{y y}=-\varepsilon_{z z}=\varepsilon$. This problem was analyzed by Harrison et al. in [29], where it was concluded that

$$
U_{\xi}(\varepsilon)=U\left(1-\lambda \varepsilon^{2}\right),
$$

for some constant $\lambda$.

Repeating the approach done for the uniaxial case, in this situation the dielectric tensor change, up to first order in $\varepsilon$, takes the form

$$
\delta\left(\overline{\boldsymbol{\epsilon}}^{-1}\right)=-\frac{2 d^{2} e^{2} \gamma}{3 \epsilon_{0} v_{c} n^{4} U}\left(\begin{array}{ccc}
0 & 0 & 0 \\
0 & 1 & 0 \\
0 & 0 & -1
\end{array}\right) \varepsilon .
$$

The corresponding dielectric matrix expected from the photoelastic coefficients is

$$
\delta\left(\overline{\boldsymbol{\epsilon}}^{-1}\right)=\left(\begin{array}{ccc}
0 & 0 & 0 \\
0 & p_{11}-p_{12} & 0 \\
0 & 0 & -\left(p_{11}-p_{12}\right)
\end{array}\right) \varepsilon .
$$

Once again we see that the shape of the last two matrices match and that the model is consistent with the photoelastic properties of the crystal. Furthermore, the value of $p_{11}-p_{12}$ can be directly evaluated from Eq. (57) and the results are shown in the last column of Table I. Once again, the calculated results are very close to the real values for $p_{11}-p_{12}$, thus showing very good agreement with experiment, except for Ge. In this case the relative error is higher, but the absolute difference between the experimental and calculated values is within the experimental error in Ref. [43].

The previous analysis shows that our approach to strain effects in silicon is consistent with a first order theory (the photoelastic effect), both numerically and phenomenologically.

\section{B. Physical properties of strain-induced $\chi^{(2)}$}

Since our model is consistent with a first order theory in the optical fields and strain, i.e., the photoelastic effect, we shall now apply it to strain-induced $\chi^{(2)}$ and verifying that it satisfies the properties that have been validated experimentally. For that, we will apply our model to a practical device in order to take conclusions about the properties of the generated $\chi^{(2)}$.

Consider the waveguide shown in Fig. 4(a), which is the structure usually used in strained silicon devices towards Pockels effect modulation $[5,6,8]$. In this case, the waveguide coordinates $\{\hat{\boldsymbol{x}}, \hat{\boldsymbol{y}}, \hat{\boldsymbol{z}}\}$ are given in terms of the crystal coordinates $\{\hat{\mathbf{1}}, \hat{\mathbf{2}}, \hat{\mathbf{3}}\}$ by

$$
\begin{aligned}
& \hat{\boldsymbol{x}}=\frac{1}{\sqrt{2}}(\hat{\mathbf{1}}+\hat{\mathbf{2}}) \\
& \hat{\boldsymbol{y}}=\hat{\mathbf{3}} \\
& \hat{z}=\frac{1}{\sqrt{2}}(\hat{\mathbf{1}}-\hat{\mathbf{2}}) .
\end{aligned}
$$

The straining layer placed on top of the waveguide has an initial stress $\sigma_{0}$, which will induce a strain field $\overline{\boldsymbol{\varepsilon}}(\boldsymbol{r})$ in the waveguide. Since the waveguide extends over the $z$ direction, the $z$ strain/strain gradients components can be neglected. In addition, we will neglect their contribution in our analysis. As 
(a)

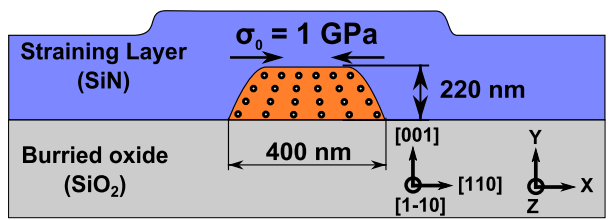

(b)
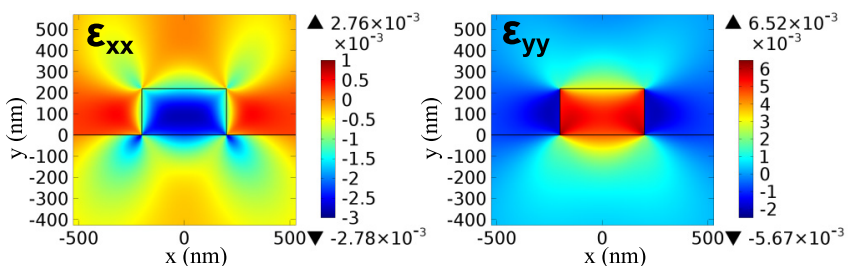

(c)

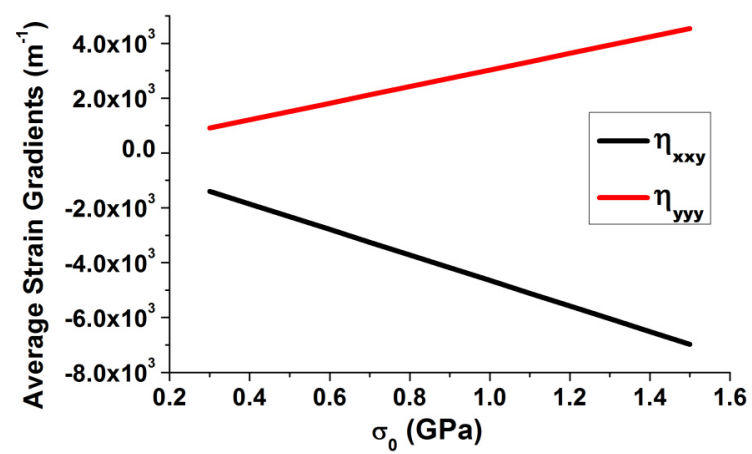

FIG. 4. (a) Cross section of the strained silicon device under consideration. (b) Principal strain components $\varepsilon_{x x}(x, y)$ and $\varepsilon_{y y}(x, y)$ generated by the strain overlayer with $\sigma_{0}=1 \mathrm{GPa}$. (c) Linear relation between the average strain gradients components $\eta_{x x y}$ and $\eta_{y y y}$ in the waveguide and the initial stress $\sigma_{0}$.

referred to in Sec. II.3.1, our model is only applicable to the principal components of strain $\varepsilon_{i i}$. This is not very limiting as our simulations show that the shear-strain components inside the Si waveguide are much smaller than the principal ones $\varepsilon_{i i}$, so we may neglect their contribution without much loss of generality. Therefore, we are only left with the components $\eta_{x x x}, \eta_{y y x}, \eta_{x x y}$, and $\eta_{y y y}$.

The strain-induced bond polarities [Eq. (37)] in the waveguide coordinates, are calculated after rewriting the bond vector coordinates [Eq. (40)] in the $\{\hat{\boldsymbol{x}}, \hat{\boldsymbol{y}}, \hat{\boldsymbol{z}}\}$ laboratory basis. The $\chi^{(2)}$ components in the laboratory coordinates can be extracted after replacing the polarities $\sigma_{\xi}$ into Eqs. (41)-(43) for the macroscopic polarization, in the laboratory coordinates. After these calculations are performed, the relevant $\chi^{(2)}$ components for this device become:

$$
\begin{gathered}
\chi_{x x x}^{(2)}=\frac{2 d^{6} K}{27 \epsilon_{0}}\left[2(\beta-\alpha) \eta_{x x x}+(3 \beta-\alpha) \eta_{y y x}\right] \\
\chi_{y y x}^{(2)}=\chi_{x x x}^{(2)} \\
\chi_{x x y}^{(2)}=\frac{2 d^{6} K}{27 \epsilon_{0}}\left[2(\beta-\alpha) \eta_{x x y}+(3 \beta-\alpha) \eta_{y y y}\right] \\
\chi_{y y y}^{(2)}=\frac{d^{6} K}{27 \epsilon_{0}}[3 \beta-\alpha)\left(\eta_{x x y}+\eta_{y y y}\right] .
\end{gathered}
$$

This set of equations gives us all the required information about the $\chi^{(2)}$ tensor in any point of space in terms of the strain gradients in that same point. We can now compare this result with the main claims on strain-induced $\chi^{(2)}$ in silicon.

We start by noticing that the previous equations have the form

$$
\chi_{i j k}^{(2)}=\sum_{l m n} \Gamma_{i j k, l m n} \eta_{l m n}
$$

which is a linear combination of strain gradients, as previously suggested in Refs. [17,44]. Moreover, from our model the coefficients $\Gamma_{i j k, l m n}$ are known and depend only on $\alpha$ and $\beta$. For instance, from Eq. (61) we extract

$$
\Gamma_{x x y, x x y}=\frac{4 d^{6} K}{27 \epsilon_{0}}(\beta-\alpha), \quad \Gamma_{x x y, y y y}=\frac{2 d^{6} K}{27 \epsilon_{0}}(3 \beta-\alpha)
$$

and this can be done to any coefficient $\Gamma_{i j k, l m n}$, always in terms of only $\alpha$ and $\beta$. This is in line with the claims that $\chi^{(2)}$ should be proportional to strain gradients and not to strain itself, as it has been suggested in many publications in the past years [6-9,17]; more importantly it gives the exact value of the weight of each strain gradient direction for the desired $\chi^{(2)}$ component. Another known experimental fact of strained silicon is that $\chi^{(2)}$ has a linear relationship with the initial stress $\sigma_{0}$ in the straining layer $[10,12,45]$.

In Fig. 4(c), we see the simulation of the average $\eta_{x x y}$ and $\eta_{y y y}$ in the waveguide for different values of $\sigma_{0}$, and it is clear the linear relationship between these two quantities. This is true for any $\eta_{i j k}$ component. Since $\chi^{(2)}$ is linear with $\eta_{i j k}$, it is straightforward to conclude that, regardless of the values of $\alpha$ and $\beta$, our model predicts

$$
\chi^{(2)} \propto \sigma_{0},
$$

which is coherent with the experimental data in Ref. [45].

\section{Estimation of the order of magnitude of $\chi^{(2)}$}

As can be seen from Eqs. (59)-(62), $\chi^{(2)}$ depends strongly on the parameters $\alpha$ and $\beta$, defined in Eq. 36. To determine these two parameters, the best approach would be to use the common practice in any semiempirical model and fit the experimental data to the theory. This would allow to extract the values of $\alpha$ and $\beta$ that give the best fit for a particular centrosymmetric material.

However, as already mentioned, all the quantitative values of strain-induced $\chi^{(2)}$ in silicon published in the literature, in particular those in Refs. [5-8] have strong parasitic contributions from carriers $[16,18,19]$. Consequently, no reliable numerical data for $\chi^{(2)}$ in strained silicon is available right now to allow for a confident fit of $\alpha$ or $\beta$ and their estimation must be done by approaching the definition in Eq. (36).

The evaluation of the integral in Eq. (33) is not a simple task, not only because it is a difficult integral to evaluate, but mainly because the real form of the silicon crystal potential $V(\boldsymbol{r})$ must be entirely known. The potential $V(\boldsymbol{r})$ is recognized to be difficult to be known exactly [38], so any result deduced from $V(\boldsymbol{r})$ will always have associated errors. Nevertheless, we will seek for an estimate of the order of magnitude of $\chi^{(2)}$ in Eqs. (59)-(62) and then compare with the most recent experimental results on strained silicon and comment on the result. 
To evaluate the order of magnitude of $\boldsymbol{\theta}_{i}^{\xi}$, we must simplify the integral in Eq. (33). For that we make the approximation $\left\|\boldsymbol{r}-\boldsymbol{R}_{A_{i}}\right\| \sim\left\|\boldsymbol{R}_{A}-\boldsymbol{R}_{A_{i}}\right\|=d$, which basically means that the hybrid wave function $h_{\xi}^{A}(\boldsymbol{r})$ is considered to be strong only close to the original atom. Despite not being entirely true because the hybrid extends along its bond, this simplification should not change considerably the order of magnitude of the integral of Eq. (33). In that case, Eq. (35) becomes

$$
\begin{aligned}
\boldsymbol{\theta}_{i}^{\xi} & \left.\sim \frac{\partial V}{\partial r}\right|_{d} \frac{1}{d} \int\left|h_{\xi}^{A}(\boldsymbol{r})\right|^{2} \cdot\left(\boldsymbol{r}-\boldsymbol{R}_{A_{i}}\right) d V \\
& \left.\sim \frac{1-\gamma}{2 d} \cdot \frac{\partial V}{\partial r}\right|_{d} \boldsymbol{\xi}-\left.\frac{1}{d} \frac{\partial V}{\partial r}\right|_{d} \boldsymbol{\xi}_{i} .
\end{aligned}
$$

The previous equation gives us values for $\alpha$ and $\beta$ which are merely approximations, but should be in the same order of magnitude of the real ones:

$$
\left.\alpha \sim \frac{1-\gamma}{2 d} \cdot \frac{\partial V}{\partial r}\right|_{d}, \quad \beta \sim-\left.\frac{1}{d} \frac{\partial V}{\partial r}\right|_{d}
$$

As already mentioned, the determination of the real $\mathrm{Si}$ crystal potential $V(\boldsymbol{r})$ is a very complex problem, which has been studied for many years [46-50]. Because of its complexity, in this paper we will only compare the numerical results for two crystal potentials. The first one is the simplest Coulomb potential generated by a $\mathrm{Si}^{4+}$ ion

$$
V_{C}(\boldsymbol{r})=-\kappa \frac{4}{r}
$$

with $\kappa=e^{2} /\left(4 \pi \epsilon_{0}\right)=2.3 \times 10^{-28} \mathrm{~kg} \mathrm{~m}^{3} / \mathrm{s}^{2}$. The second is a local pseudopotential $V_{P S}(r)$ developed by Huang et al. in Ref. [50] using the modern tools of density functional theory for the Si crystal. The explicit form of $V_{P S}(r)$ around $r=d=0.235 \mathrm{~nm}$ was obtained by fitting that region of the corresponding curve in Fig. 3 of Ref. ([50]) with an analytical function.

We apply this to Eq. (68) for both of these potentials, taking $d=0.235 \mathrm{~nm}$ and focusing only on the $\chi_{x x y}^{(2)}$ component (which is the one that has been more strongly studied in the literature [4-8]), we get for the $V_{C}$ and $V_{P S}$ potentials, respectively (in S.I. units):

$$
\chi_{x x y_{C}}^{(2)} \sim 8.0 \times 10^{-17}\left(\mathrm{~m}^{2} / \mathrm{V}\right) \eta_{y y y}+4.6 \times 10^{-17}\left(\mathrm{~m}^{2} / \mathrm{V}\right) \eta_{x x y}
$$

$\chi_{x x y_{P S}}^{(2)} \sim 1.9 \times 10^{-16}\left(\mathrm{~m}^{2} / \mathrm{V}\right) \eta_{y y y}+1.1 \times 10^{-16}\left(\mathrm{~m}^{2} / \mathrm{V}\right) \eta_{x x y}$.

Now, by using the values $\eta_{x x y} \simeq-2 \times 10^{4} \mathrm{~m}^{-1}$ and $\eta_{y y y} \simeq$ $10^{4} \mathrm{~m}^{-1}$ which are the values presented in Fig. 5 at the edge of the waveguide (where the applied electric field for Pockels effect is stronger and thus more relevant, as shown in Ref. [19]), we get:

$$
\chi_{x x y_{C}}^{(2)} \sim 0.1 \mathrm{pm} / \mathrm{V}, \quad \chi_{x x y P S}^{(2)} \sim 0.3 \mathrm{pm} / \mathrm{V} .
$$

The dependence of $\chi^{(2)}$ on the choice of potential $V(\boldsymbol{r})$ is clear from the results above. It means that any difference between predicted and experimental results can be attributed to a limitation of the potential $V(\boldsymbol{r})$ to describe the crystal phenomena. This problem can only be overcome by using a

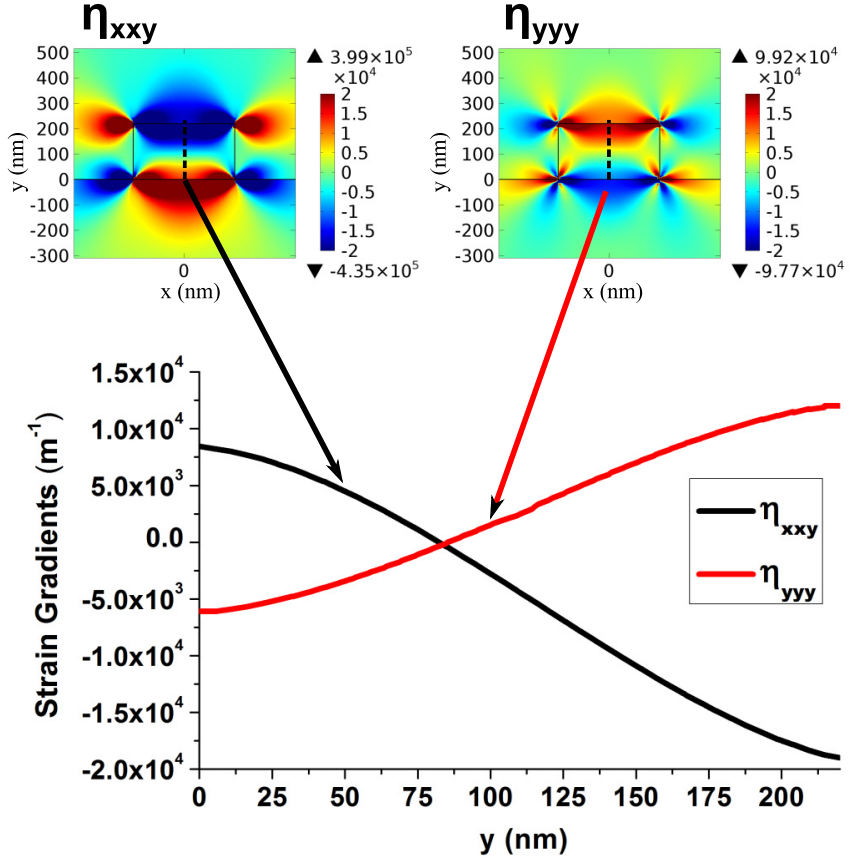

FIG. 5. Strain gradients components $\eta_{x x y}(x, y)$ and $\eta_{y y y}(x, y)$ distribution with $\sigma_{0}=1 \mathrm{GPa}$ and the corresponding values over the vertical dashed black line throughout the center of the waveguide.

semiempirical method and fit $\alpha$ and $\beta$ to available experimental data. Any other way of obtaining these two parameters will inevitably have errors associated because the used potential $V(\boldsymbol{r})$ will always be an approximation to the much more complex potential felt by the electrons in a real crystal.

On the other hand, it is not straightforward which published experimental values we should compare it to. It is now widely accepted that previously reported values of $\chi^{(2)}$ on the order of magnitude of $100 \mathrm{pm} / \mathrm{V}$ like the ones published in Refs. $[5,6,8]$ were wrongly interpreted and they are mainly due to free carriers and not to strain. In fact, latest results, which account for these effects, suggest values of $\chi^{(2)}$ much lower than these and their order of magnitude should be around $10 \mathrm{pm} / \mathrm{V}$ [16] or even as low as $1 \mathrm{pm} / \mathrm{V}$ [51]. Moreover, even these lower values of $\chi^{(2)}$ could have been erroneously estimated as latest publications suggest that the electric field applied to the waveguide to induce Pockels effect modulation is not homogeneous throughout the waveguide, but strongly modified by carriers effects [19], which does not seem to have been taken into account in these publications.

We would like to point out that the main purpose of this paper is to develop a phenomenological model to describe the dependence of the $\chi^{(2)}$ components on the different directions of strain, rather than getting an exact estimate of the numerical predictions. Nevertheless, the obtained values by our model in Eq. (72) are closer to the order of magnitude of the latest experimental results available on strain-induced $\chi^{(2)}$ [51] than any previous models published on the subject [13].

\section{CONCLUSION}

In this paper we develop and present an atomistic model, based on the bond orbital model to describe the second order 
nonlinear effects generated by strain in the silicon crystal. This model gives a spatial quantitative and well defined relation between the $\chi^{(2)}$ tensor and the strain tensor $\overline{\boldsymbol{\varepsilon}}$.

We have shown that $\chi^{(2)}$ is proportional to a weighted sum of strain gradient components, as suggested by many publications. The weighting coefficients depend only on two coefficients, $\alpha$ and $\beta$, which can be theoretically estimated but should be experimentally determined to fully validate this model.

By applying this model to the first-order phenomenon of photoelasticity, we confirmed the consistency between our theory and the properties of general cubic crystals, showing very good physical and numerical agreement with the experimentally determined photoelastic coefficients for $\mathrm{C}, \mathrm{Si}$, and $\mathrm{Ge}$ crystals. This validation of the first order approach gives high credibility to our second-order theory on strain-induced $\chi^{(2)}$.

Finally, we investigated the second-order effects in a specific geometry, and we were able to show agreement of our model with the known properties of strain-induced $\chi^{(2)}$ in silicon. Furthermore, we estimated the order of magnitude of a component of $\chi^{(2)}$ calculated using our model, and its values (between $0.1 \mathrm{pm} / \mathrm{V}$ and $0.3 \mathrm{pm} / \mathrm{V}$ ) are close to the experimental order of magnitude of the latest published experimental results. Nevertheless, this value can be strongly improved once reliable experimental data is available for a confident fit of the numerical predictions of this model.

We consider that the presented model is of extreme relevance for the study of nonlinear effects in strained silicon photonics. With the relation between $\chi^{(2)}$ and $\overline{\boldsymbol{\varepsilon}}$ that we developed in this paper, the optimization of strained silicon devices is finally possible. The strain distribution in the crystal can be engineered to maximize the most relevant $\chi^{(2)}$ components for the desired device, and this opens a whole new route towards the improvement of nonlinear effects in strained silicon, bringing us closer to high performance devices based on this kind of effect.

\section{ACKNOWLEDGMENTS}

Authors would like to thank Xavier Le Roux from IEF and Frédéric Boeuf from STMicroelectronics (Crolles, France) for fruitful discussions. The authors also acknowledge STMicroelectronics for the financial support of the P. Damas' scholarship. This project has received funding from the European Research Council (ERC) under the European Union's Horizon 2020 research and innovation program (ERC POPSTAR Grant No. 647342).
[1] J. M. Fedeli, L. Di Cioccio, D. Marris-Morini, L. Vivien, R. Orobtchouk, P. Rojo-Romeo, C. Seassal, and F. Mandorlo, Adv. Optical Technol. 2008, 412518 (2008).

[2] J. Leuthold, C. Koos, and W. Freude, Nat. Photon. 4, 535 (2010).

[3] G. T. Reed, G. Mashanovich, F. Y. Gardes, and D. J. Thomson, Nat. Photon. 4, 518 (2010).

[4] R. S. Jacobsen, K. N. Andersen, P. I. Borel, J. Fage-Pedersen, L. H. Frandsen, O. Hansen, M. Kristensen, A. V. Lavrinenko, G. Moulin, H. Ou, C. Peucheret, B. Zsigri, and A. Bjarklev, Nature (London) 441, 199 (2006).

[5] B. Chmielak, M. Waldow, C. Matheisen, C. Ripperda, J. Bolten, T. Wahlbrink, M. Nagel, F. Merget, and H. Kurz, Opt. Express 19, 17212 (2011).

[6] B. Chmielak, C. Matheisen, C. Ripperda, J. Bolten, T. Wahlbrink, M. Waldow, and H. Kurz, Opt. Express 21, 25324 (2013).

[7] M. W. Puckett, J. S. T. Smalley, M. Abashin, A. Grieco, and Y. Fainman, Opt. Lett. 39, 1693 (2014).

[8] P. Damas, X. Le Roux, D. Le Bourdais, E. Cassan, D. MarrisMorini, N. Izard, T. Maroutian, P. Lecoeur, and L. Vivien, Opt. Express 22, 22095 (2014).

[9] M. Cazzanelli, F. Bianco, E. Borga, G. Pucker, M. Ghulinyan, E. Degoli, E. Luppi, V. Véniard, S. Ossicini, D. Modotto, S. Wabnitz, R. Pierobon, and L. Pavesi, Nat. Mater. 11, 148 (2012).

[10] C. Schriever, C. Bohley, J. Schilling, and R. B. Wehrspohn, Materials 5, 889 (2012).

[11] S. V. Govorkov, V. I. Emel'yanov, N. I. Koroteev, G. I. Petrov, I. L. Shumay, V. V. Yakovlev, and R. V. Khokhlov, J. Opt. Soc. Am. B 6, 1117 (1989).

[12] J. Huang, Jpn. J. Appl. Phys. 33, 3878 (1994).

[13] N. N. K. Hon, K. K. K. Tsia, D. R. D. Solli, B. Jalali, and J. B. Khurgin, in 2009 6th IEEE International Conference on Group IV Photonics (IEEE, San Francisco, CA, 2009), pp. 232-234.
[14] N. K. Hon, K. K. Tsia, D. R. Solli, and B. Jalali, Appl. Phys. Lett. 94, 091116 (2009).

[15] E. Luppi, E. Degoli, M. Bertocchi, S. Ossicini, and V. Véniard, Phys. Rev. B 92, 075204 (2015).

[16] C. Schriever, F. Bianco, M. Cazzanelli, M. Ghulinyan, C. Eisenschmidt, J. de Boor, A. Schmid, J. Heitmann, L. Pavesi, and J. Schilling, Adv. Opt. Mater. 3, 129 (2015).

[17] C. L. Manganelli, P. Pintus, and C. Bonati, Opt. Express 23, 28649 (2015).

[18] S. S. Azadeh, F. Merget, M. P. Nezhad, and J. Witzens, Opt. Lett. 40, 1877 (2015).

[19] R. Sharma, M. W. Puckett, H.-H. Lin, F. Vallini, and Y. Fainman, Appl. Phys. Lett. 106, 241104 (2015).

[20] J. Chen, Z. H. Levine, and J. W. Wilkins, Appl. Phys. Lett. 66, 1129 (1995).

[21] M. Z. Huang and W. Y. Ching, Phys. Rev. B 47, 9464 (1993).

[22] Z. H. Levine, Phys. Rev. B 49, 4532 (1994).

[23] B. Levine, Phys. Rev. B 7, 2600 (1973).

[24] W. A. Harrison, Phys. Rev. B 8, 4487 (1973).

[25] G. D. Powell, J.-F. Wang, and D. E. Aspnes, Phys. Rev. B 65, 205320 (2002).

[26] D. E. Aspnes, Phys. Status Solidi B 247, 1873 (2010).

[27] W. A. Harrison, Electronic Structure and the Properties of Solids, Dover Books on Physics (Dover Publications, New York, 1989).

[28] W. A. Harrison and S. Ciraci, Phys. Rev. B 10, 1516 (1974).

[29] W. A. Harrison and J. C. Phillips, Phys. Rev. Lett. 33, 410 (1974).

[30] B. Levine, Phys. Rev. Lett. 22, 787 (1969).

[31] W. A. Harrison, Phys. Rev. A 7, 1876 (1973).

[32] C. Huang, J. A. Moriarty, A. Sher, and R. A. Breckenridge, Phys. Rev. B 12, 5395 (1975).

[33] M. E. Lines, J. Appl. Phys. 60, 1472 (1986). 
[34] P. M. Gullett, M. F. Horstemeyer, M. I. Baskes, and H. Fang, Modell. Simul. Mater. Sci. Eng. 16, 015001 (2008).

[35] A. Stukowski and A. Arsenlis, Modell. Simul. Mater. Sci. Eng. 20, 035012 (2012).

[36] P. H. Mott, A. S. Argon, and U. W. Suter, J. Comput. Phys. 101, 140 (1992).

[37] L. D. Landau and E. M. Lifshitz, The Theory of Elasticity, Vol. 7 (Pergamon Press, Oxford, 1970).

[38] J. Witzens, Comput. Phys. Commun. 185, 2221 (2014).

[39] Z. H. Levine, H. Zhong, S. Wei, D. C. Allan, and J. W. Wilkins, Phys. Rev. B 45, 4131 (1992).

[40] M. H. Grimsditch and A. K. Ramdas, Phys. Rev. B 11, 3139 (1975).

[41] L. S. Hounsome, R. Jones, M. J. Shaw, and P. R. Briddon, Phys. Status Solidi A 203, 3088 (2006).

[42] M. Huang, Int. J. Solids Struct. 40, 1615 (2003).

[43] A. Feldman, R. M. Waxler, and D. Horowitz, J. Appl. Phys. 49, 2589 (1978).
[44] P. Damas, X. Le Roux, E. Cassan, N. Izard, D. Marris-morini, A. Bosseboeuf, P. Lecoeur, T. Maroutian, and L. Vivien, in Asia Communications and Photonics Conference 2013, OSA Technical Digest (online) (Optical Society, Washington, 2013), paper AF2B.8.

[45] C. Schriever, C. Bohley, and R. B. Wehrspohn, Opt. Lett. 35, 273 (2010).

[46] J. A. Appelbaum and D. R. Hamann, Phys. Rev. B 8, 1777 (1973).

[47] P. W. Anderson, Phys. Rev. 181, 25 (1969).

[48] J. R. Chelikowsky, K. M. Glassford, and J. C. Phillips, Phys. Rev. B 44, 1538 (1991).

[49] H. Wendel and R. M. Martin, Phys. Rev. Lett. 40, 950 (1978).

[50] C. Huang and E. a. Carter, Phys. Chem. Chem. Phys. 10, 7109 (2008).

[51] M. Borghi, M. Mancinelli, F. Merget, J. Witzens, M. Bernard, M. Ghulinyan, G. Pucker, and L. Pavesi, Opt. Lett. 40, 5287 (2015). 